\title{
Formation of sharp eccentric rings in debris disks with gas but without planets
}

\author{
W. Lyra ${ }^{1,2,3} \&$ M. Kuchner ${ }^{4}$
}

\begin{abstract}
'Debris disks' around young stars (analogues of the Kuiper Belt in our Solar System) show a variety of non-trivial structures attributed to planetary perturbations and used to constrain the properties of those planets ${ }^{1-3}$. However, these analyses have largely ignored the fact that some debris disks are found to contain small quantities of gas $^{4-9}$, a component that all such disks should contain at some level ${ }^{10,11}$. Several debris disks have been measured with a dust-to-gas ratio of about unity ${ }^{4-9}$, at which the effect of hydrodynamics on the structure of the disk cannot be ignored ${ }^{12,13}$. Here we report linear and nonlinear modelling that shows that dust-gas interactions can produce some of the key patterns attributed to planets. We find a robust clumping instability that organizes the dust into narrow, eccentric rings, similar to the Fomalhaut debris disk $^{14}$. The conclusion that such disks might contain planets is not necessarily required to explain these systems.
\end{abstract}

Disks around young stars seem to pass through an evolutionary phase when the disk is optically thin and the dust-to-gas ratio $\varepsilon$ ranges from 0.1 to 10 . The nearby stars $\beta$ Pictoris ${ }^{5,6,15-17}$, HD32297 (ref. 7), 49 Ceti (ref. 4) and HD 21997 (ref. 9) all host dust disks resembling ordinary debris disks and also have stable circumstellar gas detected in molecular CO, $\mathrm{Na}$ I or other metal lines; the inferred mass of gas ranges from lunar masses to a few Earth masses (Supplementary Information). The gas in these disks is thought to be produced by planetesimals or dust grains themselves, by means of sublimation, photodesorption ${ }^{10}$ or collisions ${ }^{11}$, processes that should occur in every debris disk at some level.

Structures may form in these disks by a recently proposed instability ${ }^{12,13}$. Gas drag causes dust in a disk to concentrate at pressure maxima ${ }^{18}$; however, when the disk is optically thin to starlight, the gas is most probably primarily heated by the dust, by photoelectric heating. In this circumstance, a concentration of dust that heats the gas creates a local pressure maximum that in turn can cause the dust to concentrate more. The result of this photoelectric instability could be that the dust clumps into rings or spiral patterns or other structures that could be detected by coronographic imaging or other methods.

Indeed, images of debris disks and transitional disks show a range of asymmetries and other structures that call for explanation. Traditionally, explanations for these structures rely on planetary perturbers-a tantalizing possibility. However, so far it has been difficult to prove that these patterns are clearly associated with exoplanets ${ }^{19,20}$.

Previous investigations of hydrodynamical instabilities in debris disks neglected a crucial aspect of the dynamics: the momentum equations for the dust and gas. Equilibrium terminal velocities are assumed between time steps in the numerical solution, and the dust distribution is updated accordingly. The continuity equation for the gas is not solved; that is, the gas distribution is assumed to be time-independent, despite heating, cooling, and drag forces. Moreover, previous investigations considered only one-dimensional models, which can only investigate azimuthally symmetrical ring-like patterns. This limitation also left open the possibility that, in higher dimensions, the power in the instability might collect in higher azimuthal wavenumbers, generating only unobservable clumps.
We present simulations of the fully compressible problem, solving for the continuity, Navier-Stokes and energy equations for the gas, and the momentum equation for the dust. Gas and dust interact dynamically through a drag force, and thermally through photoelectric heating. These are parametrized by a dynamical coupling time $\tau_{\mathrm{f}}$ and a thermal coupling time $\tau_{\mathrm{T}}$ (Supplementary Information). The simulations are performed with the Pencil Code ${ }^{21-24}$, which solves the hydrodynamics on a grid. Two numerical models are presented: a three-dimensional box embedded in the disk that co-rotates with the flow at a fixed distance from the star; and a two-dimensional global model of the disk in the inertial frame. In the former the dust is treated as a fluid, with a separate continuity equation. In the latter the dust is represented by discrete particles with position and velocities that are independent of the grid.

We perform a stability analysis of the linearized system of equations that should help interpret the results of the simulations (Supplementary Information). We plot in Fig. 1a-c the three solutions that show linear growth, as functions of $\varepsilon$ and $n=k H$, where $k$ is the radial wavenumber and $H$ is the gas scale height $\left(H=c_{\mathrm{s}} / \sqrt{\gamma} \Omega_{\mathrm{K}}\right.$, where $c_{\mathrm{s}}$ is the sound speed, $\Omega_{\mathrm{K}}$ the Keplerian rotation frequency and $\gamma$ the adiabatic index). The friction time $\tau_{\mathrm{f}}$ is assumed to be equal to $1 / \Omega_{\mathrm{K}}$. The left and middle panels show the growth and damping rates. The right panels show the oscillation frequencies. There is no linear instability for $\varepsilon \geq 1$ or $n \leq 1$. At low dust load and high wavenumber the three growing modes appear. The growing modes shown in Fig. 1a have zero oscillation frequency, characterizing a true instability. The two other growing solutions (Fig. 1b, c) are overstabilities, given the associated non-zero oscillation frequencies. The pattern of larger growth rates at large $n$ and low $\varepsilon$ invites us to take $\tilde{\zeta}=\varepsilon n^{2}$ as characteristic variable and to explore the behaviour of $\tilde{\zeta} \gg 1$. The solutions in this approximation are plotted in Fig. 1f, g. The instability (red) has a growth rate of roughly $0.26 \Omega_{\mathrm{K}}$ for all $\tilde{\zeta}$. The overstability (yellow) reaches an asymptotic growth rate of $\Omega_{\mathrm{K}} / 2$, at ever-growing oscillation frequencies. Damped oscillations (blue) occur at a frequency close to the epicyclic frequency.

Whereas the inviscid solution has growth even for very small wavelengths, viscosity will cap power at this regime, leading to a finite fastestgrowing mode (Supplementary Information), which we reproduce numerically (Fig. 1h). Although there is no linear growth for $\varepsilon \geq 1$, we show that there exists nonlinear growth for $\varepsilon=1$. We show in Fig. 1i the time evolution of the maximum dust surface density $\Sigma_{\mathrm{d}}$ (normalized by its initial value, $\Sigma_{0}$ ). A qualitative change in the behaviour of the system (a bifurcation) occurs when the noise amplitude of the initial velocity $\left(u_{\mathrm{rms}}\right)$ is raised far enough, as expected from nonlinear instabilities ${ }^{25,26}$. We emphasize this result because, depending on the abundance of $\mathrm{H}_{2}$, the range of $\varepsilon$ in debris disks spans both the linear and nonlinear regimes. The parameter space of $\tau_{\mathrm{T}}$ and $\tau_{\mathrm{f}}$ is explored in one-dimensional models in Supplementary Information, showing robustness.

In Fig. 2 we show the linear development and saturation of the photoelectric instability in a vertically stratified local box of size

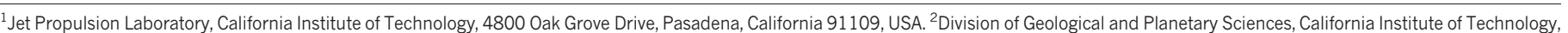
1200 E. California Boulevard MC 150-21, Pasadena, California 91125, USA. ${ }^{3}$ Department of Astrophysics, American Museum of Natural History, 79th Street at Central Park West, New York, New York 10024, USA. ${ }^{4}$ NASA Goddard Space Flight Center, Exoplanets and Stellar Astrophysics Laboratory, Code 667, Greenbelt, Maryland 21230, USA. 


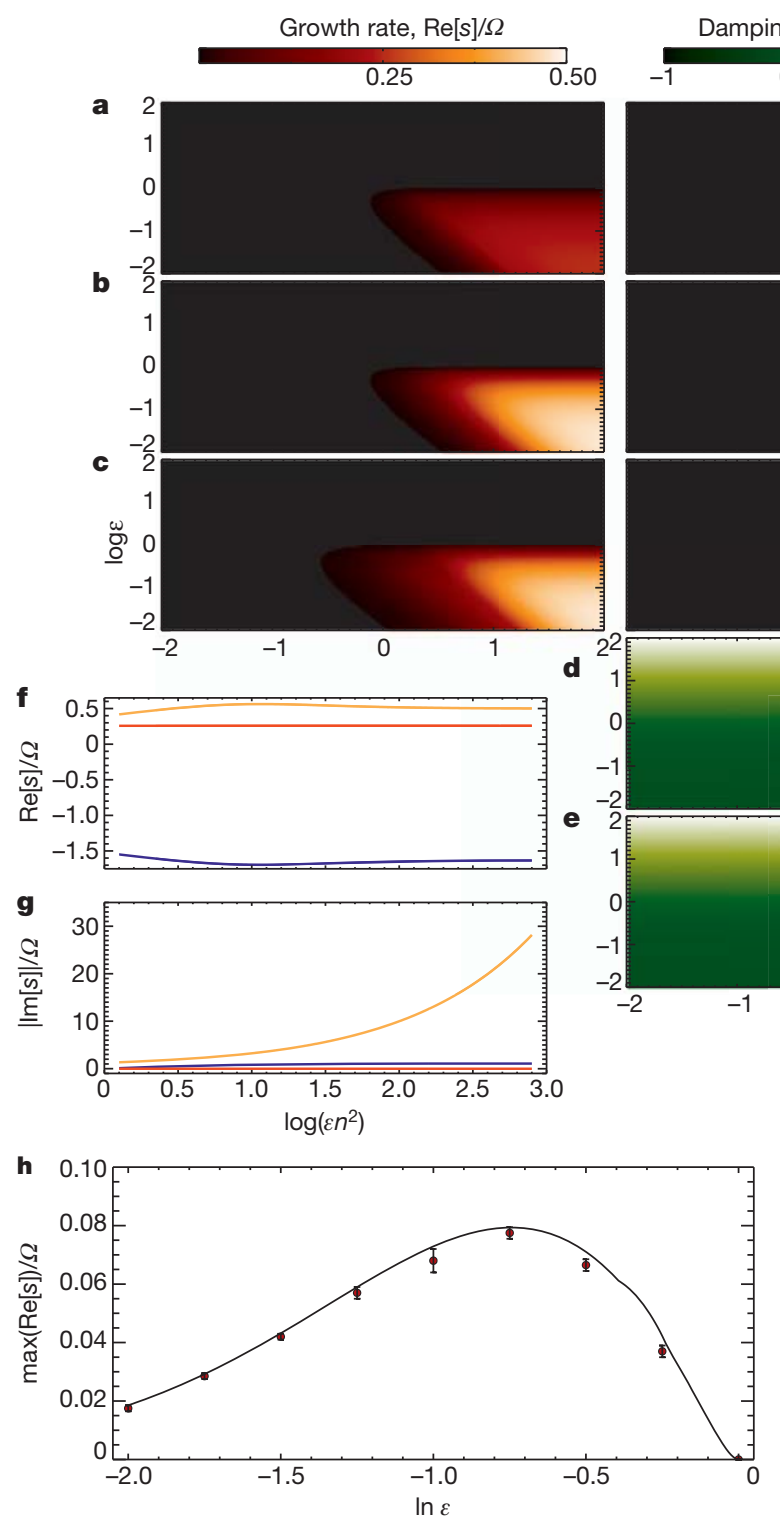

Figure $1 \mid$ Linear analysis of the axisymmetric modes of the photoelectric instability. Solutions for axisymmetric perturbations $\psi^{\prime}=\hat{\psi} \exp (s t+i k x)$, where $\hat{\psi}$ is a small amplitude, $x$ is the radial coordinate in the local Cartesian corotating frame, $k$ is the radial wavenumber, $t$ is time and $s$ is the complex frequency. Positive real $s$ means that a perturbation grows, negative $s$ indicates that a perturbation is damped, and imaginary $s$ represents oscillations. Solutions are for $\alpha=0, \tau_{\mathrm{f}}=1 / \Omega_{\mathrm{K}}$ and $\tau_{\mathrm{T}}=0$. a-e, The five solutions as functions of $n=k H$ and $\varepsilon$. Solutions a-c show linear growth. Growth is restricted to the region with low dust-to-gas ratio $(\varepsilon<1)$ and high wavenumber $(n>1)$. The growing modes in $\mathbf{b}$ and $\mathbf{c}$ have non-zero oscillation frequencies, characterizing an overstability. Conversely, solution a is a true instability. d, e, Solutions that correspond to damped oscillations through most of the parameter space. In a small region (high dust-to-gas ratio and high frequency),

$(1 \times 1 \times 0.6) H$ and resolution $255 \times 256 \times 128$. The dust and gas are initialized in equilibrium (Supplementary Information). The dust-togas ratio is given by $\log \varepsilon=-0.75$, so that there is linear instability, and viscosity $v=\alpha c_{\mathrm{s}} H$ is applied as $\alpha=10^{-4}$ (where $\alpha$ is a dimensionless parameter $^{27}$ ). The initial noise is $u_{\mathrm{rms}} / c_{\mathrm{s}}=10^{-2}$. Figure $2 \mathrm{a}$ shows the dust density $\rho_{\mathrm{d}}$ in the $x-z$ plane, and Fig. $2 \mathrm{~b}$ that in the $x-y$ plane, both at 100 orbits (the orbital period is $T_{\text {orb }}=2 \pi / \Omega_{\mathrm{K}}$ ). Figure $2 \mathrm{c}$ shows the one-dimensional $x$-dependent vertical and azimuthal average against time. Through photoelectric heating, pressure maxima are generated at the locations where dust concentrates, which in turn attract more dust by means of the drag force. There is no hint of unstable short-wavelength
Oscillation frequency, $\log (\mid \operatorname{Im}[s] / \Omega)$
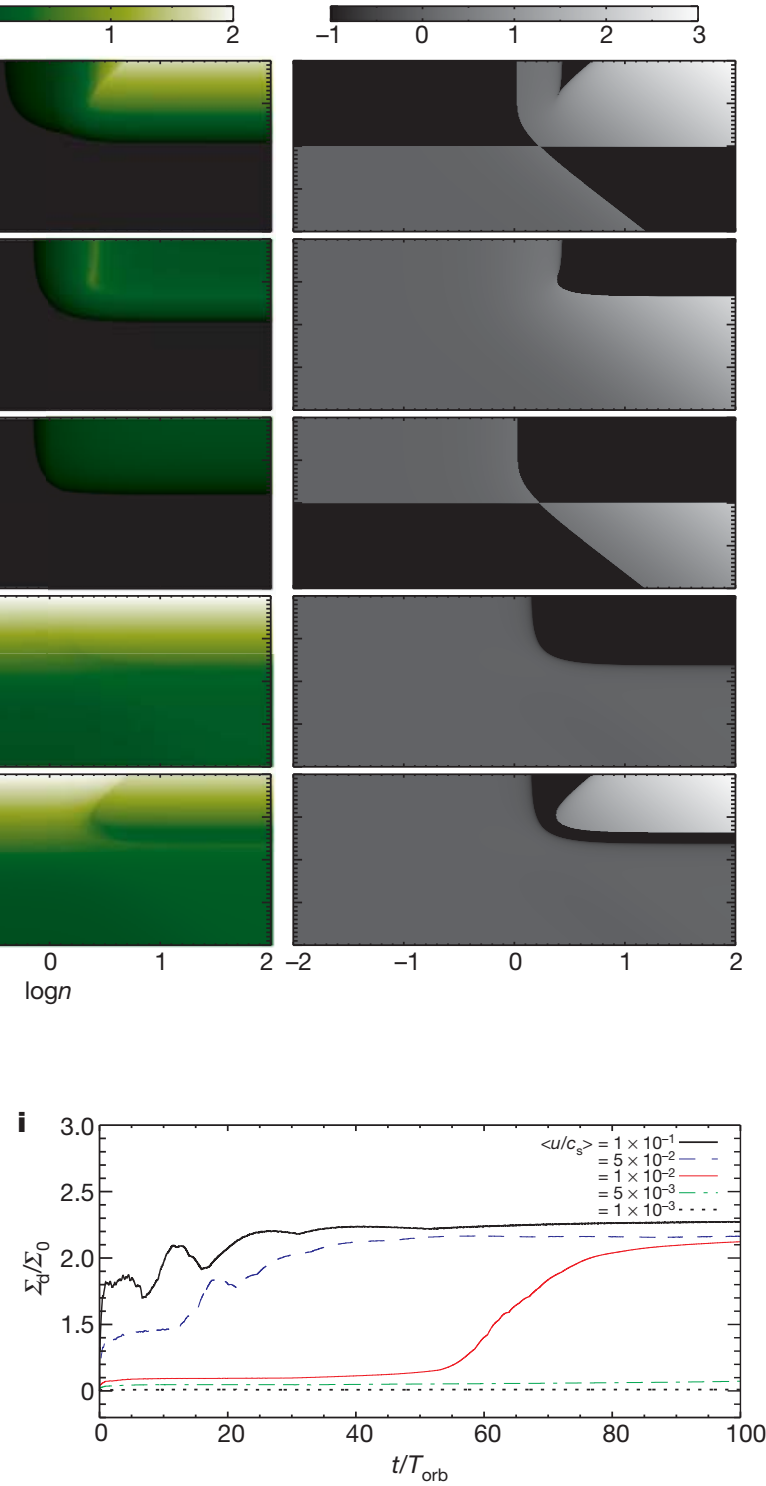

modes are exponentially damped without oscillating. $\mathbf{f}, \mathbf{g}$, Growth rate $(\mathbf{f})$ and oscillation frequency $(\mathbf{g})$. Using $\tilde{\zeta}=\varepsilon n^{2}$ and taking the limit $\tilde{\zeta} \gg 1$ permits better visualization of the three behaviours: true instability (red), overstability (yellow) and damped oscillations (blue). The other two solutions are the complex conjugate of the oscillating solutions and are not shown. $\mathbf{h}$, Growth rates. When viscosity is considered $\left(\alpha=10^{-2}\right.$ in this example), power is capped at high wavenumber, leading to a finite most-unstable wavelength. The figure shows the analytical prediction of the linear instability growth in this case (Supplementary Information) compared to the growth rates measured numerically. The overall agreement is excellent. The growth rates are only very slightly underestimated. i, Nonlinear growth. Although there is no linear instability for $\varepsilon=1$, growth occurs when the amplitude of the initial perturbation $\left(u_{\mathrm{rms}}\right)$ is increased, a hallmark of nonlinear instability.

(less than $H$ ) non-axisymmetric modes: the instability generates stripes. The simulation also shows that stratification does not quench the instability. Figure $2 \mathrm{~d}$ shows a plot of the maximum dust density against time, achieving saturation and steady state at about 70 orbits.

We consider now a two-dimensional global model. The resulting flow, in the $r-\phi$ plane ( $r$ is radius and $\phi$ is azimuth), is shown in Fig. 3a-c at selected snapshots. The flow develops into a dynamic system of narrow rings. Whereas some of the rings break into arcs, some maintain axisymmetry for the whole timespan of the simulation. It is also observed that some arcs later re-form into rings. We check that, in the absence of the drag force back-reaction, the system does not develop rings 


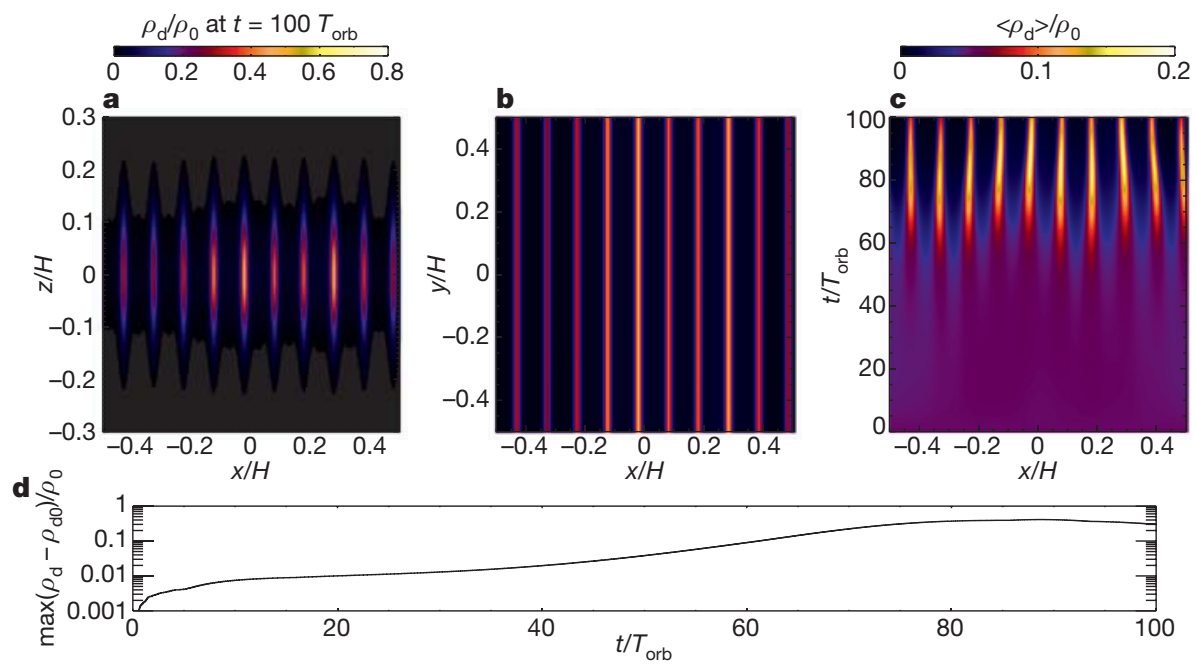

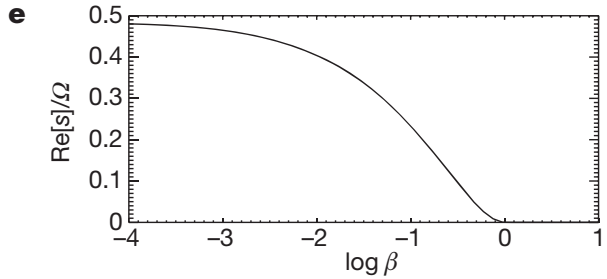

Figure $2 \mid$ Growth and saturation of the photoelectric instability. In this three-dimensional stratified local box with linearized Keplerian shear, the main source of heating is photoelectric. The equilibrium in the radial direction is between stellar gravity, Coriolis force and centrifugal force. In the vertical direction the equilibrium for the gas is hydrostatic, between stellar gravity, pressure and the drag-force back-reaction. To provide a stable stratification, an extra pressure $p_{\mathrm{b}}=\rho c_{\mathrm{b}}^{2}$ is added, where $c_{\mathrm{b}}$ is a sound speed associated with a background temperature. For the dust, a steady state is established between gravity, diffusion and drag force. The dust continually falls to the midplane but is diffused upwards. The diffusion is applied only in $z$, mimicking turbulent diffusion that is in general anisotropic. a, $x-z$ cut at $y=0$ at 100 orbits. The instability concentrates dust in a preferred wavelength. The resulting structures have stable stratification. $\mathbf{b}, x-y$ cut at the midplane $z=0$ at 100 orbits. No

(Supplementary Information). We also check that when the conditions for the streaming instability ${ }^{24}$ are considered, the photoelectric instability dominates (Supplementary Information).

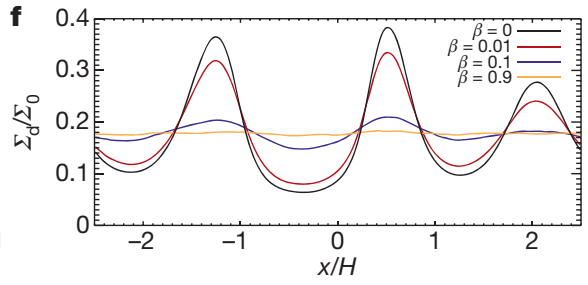

non-axisymmetric instability is observed, and the dust forms stripes. c, Time evolution of the vertically and azimuthally averaged density, showing the formation of well-defined rings. $\mathbf{d}$, Time evolution of the maximum dust density. The instability saturates at about 70 orbits in this case. The slowdown compared with the growth rate $\Omega_{\mathrm{K}} / 2$ predicted in Fig. 1 is because of the use of viscosity, and the background pressure needed for the stratification. The dimensionless parameter $\beta=\gamma\left(c_{\mathrm{b}} / c_{\mathrm{s}}\right)^{2}$ measures the strength of this term. e, Maximum growth rate, showing that linear instability exists as long as $\beta<1$. The maximum growth rate decreases smoothly from $\Omega_{\mathrm{K}} / 2$ for $\beta=0$, to zero for $\beta=1$.f. The structure formed in the dust density at $t=50$ (about eight orbits) for different values of $\beta$. At moderate values, growth still occurs at a significant fraction of the dynamical time. The run shown in a-d used $\beta=0.5$.

A development of the model is that some of the rings start to oscillate, seeming eccentric. These oscillations are epicycles in the orbital plane, with a period equalling the Keplerian, corresponding to the free
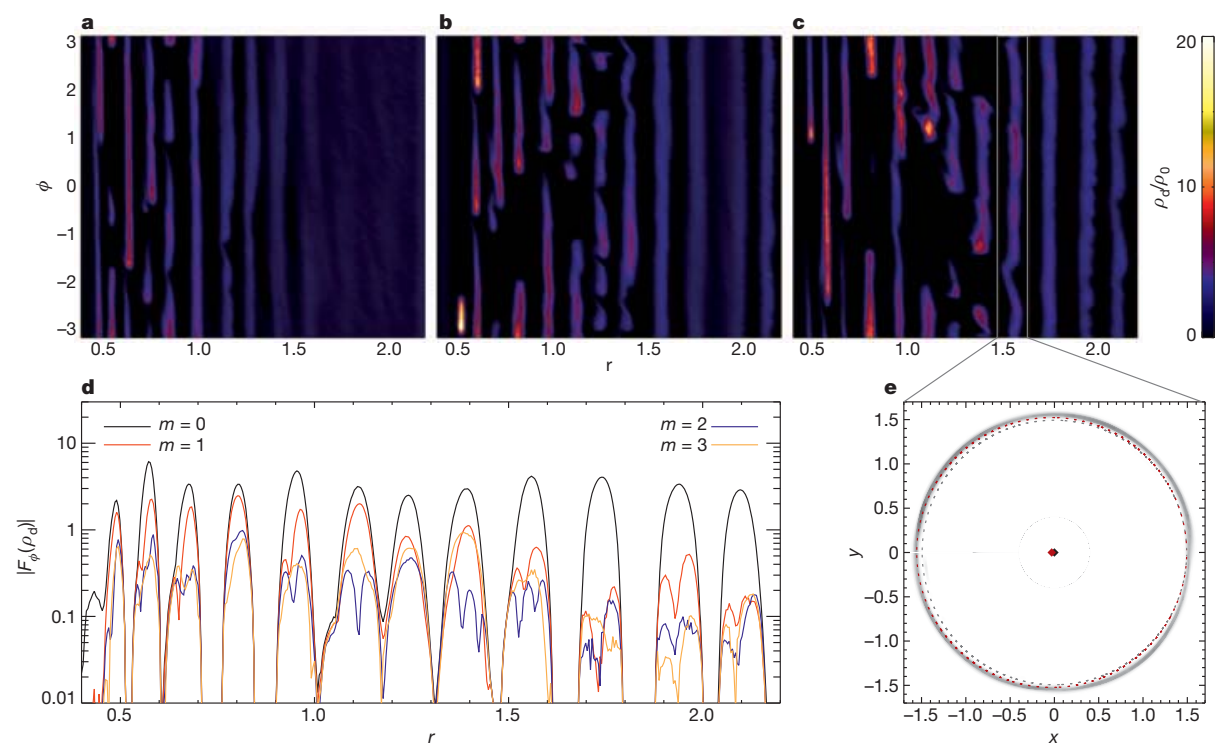

Figure 3 Sharp eccentric rings. a-c, Snapshots of the dust density in a two-dimensional global disk in polar coordinates, at 20 orbits (a), 40 orbits (b) and 60 orbits (c). The photoelectric instability initially concentrates the dust axisymmetrically into rings, at a preferred wavelength. As the simulation proceeds, some rings maintain the axisymmetry and others break into arcs. Some arcs rearrange into rings at later times, such as those at $r=0.6$ and $r=1.0$ between $\mathbf{b}$ and $\mathbf{c}$. Although mostly axisymmetric, some rings seem to oscillate, appearing off-centred or eccentric. d, We measure the azimuthal spectral power of the density shown in $\mathbf{c}$, as a function of radius. Modes from $m=0$ to $m=3$ are shown, where $m$ is the azimuthal wavenumber. e, Although the ring at $r=1.5$ has $m=0$ as the more prominent mode, we show that a circle (black dotted line) is not a good fit. An ellipse of eccentricity $e=0.03$ (red dotted line) is a better fit, although still falling short of accurately describing its shape. The black and red diamonds are the centre of the circle (the star) and the centre of the ellipse (a focal distance away from the star), respectively. 
oscillations in the right-hand side of Fig. 1a-c. We check (Supplementary Information) that they correspond to eigenvectors for which $u=v$; that is, gas and dust velocities coinciding. For this mode, the drag force and back-reaction are cancelled. So, for maintaining the eccentricity, this mode is being selected from among the other modes in the spectrum. This is naturally expected when the dust-to-gas ratio is very high. For $\varepsilon \gg 1$, the gas is strongly coupled to the dust, cancelling the gas-dust drift velocity in the same way that $\tau_{\mathrm{f}} \ll 1$ does in the opposite way, by strongly coupling the dust to the gas. In this configuration, the freely oscillating epicyclic modes can be selected.

We plot in Fig. 3e one of the oscillating rings, showing that its shape is better fitted by an ellipse (red dotted line) than by a circle (black dotted line). The eccentricity is 0.03 , which is close to the eccentricity found ${ }^{28}$ for the ring around HD $61005(e=0.045 \pm 0.015)$. We also notice that some of the clumps in Fig. 3 should become very bright in reflected light, because they have dust enhancements of an order of magnitude. In conclusion, the proposed photoelectric instability provides simple and plausible explanations for rings in debris disks, their eccentricities, and bright moving sources in reflected light.

Recent work ${ }^{29}$ suggests that the ring around Fomalhaut is confined by a pair of shepherding terrestrial-mass planets, below the current detection limits. Detection of gas around the ring would be a way to distinguish that situation from the one we propose. At present, only upper limits on the amount of gas in the Fomalhaut system exist ${ }^{30}$; however, they are relatively insensitive because they probe $\mathrm{CO}$ emission, and CO could easily be dissociated around this early A-type star.

\section{Received 27 September 2012; accepted 2 May 2013.}

1. Kuchner, M. J. \& Holman, M. J. The geometry of resonant signatures in debris disks with planets. Astrophys. J. 588, 1110-1120 (2003)

2. Chiang, E., Kite, E., Kalas, P., Graham, J. \& Clampin, M. Fomalhaut's debris disk and planet: constraining the mass of Fomalhautb from disk morphology. Astrophys. J. 693, 734-749 (2009).

3. Lagrange, A.-M. et al. A giant planet imaged in the disk of the young star $\beta$ Pictoris. Science 329, 57-60 (2010).

4. Zuckerman, B., Forveille, T. \& Kastner, J. H. Inhibition of giant-planet formation by rapid gas depletion around young stars. Nature 373, 494-496 (1995).

5. Lagrange, A. et al. The $\beta$ Pictoris circumstellar disk. XXIV. Clues to the origin of the stable gas. Astron. Astrophys. 330, 1091-1108 (1998).

6. Roberge, A., Feldman, P. D., Weinberger, A. J., Deleuil, M. \& Bouret, J.-C. Stabilization of the disk around $\beta$ Pictoris by extremely carbon-rich gas. Nature 441, 724-726 (2006).

7. Redfield, S. Gas absorption detected from the edge-on debris disk surrounding HD 32297. Astrophys. J. 656, L97-L100 (2007).

8. Maness, H. L. et al. CARMA millimeter-wave aperture synthesis imaging of the HD 32297 debris disk. Astrophys. J. 686, L25-L28 (2008).

9. Moór, A. et al. Molecular gas in young debris disks. Astrophys. J. 740, L7-L12 (2011).

10. Grigorieva, A., Thebault, P., Artymowicz, P. \& Brandeker, A. Survival of icy grains in debris discs. The role of photosputtering. Astron. Astrophys. 475, 755-764 (2007).

11. Czechowski, A. \& Mann, I. Collisional vaporization of dust and production of gas in the $\beta$ Pictoris dust disk. Astrophys. J. 660, 1541-1555 (2007).
12. Klahr, H. \& Lin, D. N. C. Dust distribution in gas disks. Il. Self-induced ring formation through a clumping instability. Astrophys. J. 632, 1113-1121 (2005).

13. Besla, $\mathrm{G}$. \& Wu, Y. Formation of narrow dust rings in circumstellar debris disks. Astrophys. J. 655, 528-540 (2007).

14. Kalas, P., Graham, J. R. \& Clampin, M. A planetary system as the origin of structure in Fomalhaut's dust belt. Nature 435, 1067-1070 (2005).

15. Olofsson, G., Liseau, R. \& Brandeker, A. Widespread atomic gas emission reveals the rotation of the $\beta$ Pictoris disk. Astrophys. J. 563, L77-L80 (2001).

16. Brandeker, A., Liseau, R., Olofsson, G. \& Fridlund, M. The spatial structure of the $\beta$ Pictoris gas disk. Astron. Astrophys. 413, 681-691 (2004).

17. Troutman, M. R., Hinkle, K. H., Najita, J. R., Rettig, T. W. \& Brittain, S. D. Ro-vibrational CO detected in the $\beta$ Pictoris circumstellar disk. Astrophys. J. 738, 12-19 (2011).

18. Takeuchi, T. \& Artymowicz, P. Dust migration and morphology in optically thin circumstellar gas disks. Astrophys. J. 557, 990-1006 (2001).

19. Janson, M. et al. Infrared non-detection of Fomalhaut b: implications for the planet interpretation. Astrophys. J. 747, 116-122 (2012).

20. Currie, T.etal. Directimaging confirmation and characterization of a dust-enshrouded candidate exoplanet orbiting Fomalhaut. Astrophys. J. 760, L32-L37 (2012).

21. Brandenburg, A. \& Dobler, W. Hydromagnetic turbulence in computer simulations. Comp. Phys. Commun. 147, 471-475 (2002).

22. Lyra, W., Johansen, A., Klahr, H. \& Piskunov, N. Global magnetohydrodynamical models of turbulence in protoplanetary disks. I. A cylindrical potential on a Cartesian grid and transport of solids. Astron. Astrophys. 479, 883-901 (2008).

23. Lyra, W., Johansen, A., Zsom, A., Klahr, H. \& Piskunov, N. Planet formation bursts at the borders of the dead zone in 2D numerical simulations of circumstellar disks. Astron. Astrophys. 497, 869-888 (2009).

24. Youdin, A. \& Johansen, A. protoplanetary disk turbulence driven by the streaming instability: linear evolution and numerical methods. Astrophys. J. 662, 613-626 (2007)

25. Stuart, J. T. Nonlinear stability theory. Annu. Rev. Fluid Mech. 3, 347-370 (1971).

26. Lesur, G. \& Papaloizou, J. C. B. The subcritical baroclinic instability in local accretion disc models. Astron. Astrophys. 513, 60-71 (2010).

27. Shakura, N. I. \& Sunyaev, R. A. Black holes in binary systems. Observational appearance. Astron. Astrophys. 24, 337-355 (1973).

28. Buenzli, E. et al. Dissecting the Moth: discovery of an off-centered ring in the HD 61005 debris disk with high-resolution imaging. Astron. Astrophys. 524, L1-L4 (2010).

29. Boley, A. C. et al. Constraining the planetary system of Fomalhaut using highresolution ALMA observations. Astrophys. J. 750, L21-L24 (2012)

30. Liseau, R. Molecular line observations of southern main-sequence stars with dust disks: $\alpha$ Ps A, $\beta$ Pic, $\varepsilon$ Eri and HR 4796 A. Does the low gas content of the $\beta$ Pic and $\varepsilon$ Eri disks hint at the presence of planets? Astron. Astrophys. 348, 133-138 (1999).

Supplementary Information is available in the online version of the paper.

Acknowledgements We thank H. Latter and G. Stewart for discussions. The writing of this paper started at the American Museum of Natural History, with financial support by the National Science Foundation under grant no. AST10-09802, and was completed at the Jet Propulsion Laboratory, California Institute of Technology, under a contract with the National Aeronautics and Space Administration. This research was supported by an allocation of advanced computing resources supported by the National Science Foundation. The computations were performed on the Kraken system at the National Institute for Computational Sciences. W.L. is a Carl Sagan fellow. M.K. is supported in part by the NASA Astrobiology Institute through the Goddard Center for Astrobiology.

Author Contributions W.L. contributed to developing the model, performed the calculations and wrote the manuscript. M.K. contributed to developing the model and writing the manuscript.

Author Information Reprints and permissions information is available at www.nature.com/reprints. The authors declare no competing financial interests. Readers are welcome to comment on the online version of the paper. Correspondence and requests for materials should be addressed to W.L. (wlyra@caltech.edu) or M.K. (marc.j.kuchner@nasa.gov). 